\title{
Extension of MINDO/3-FORCES Method
}

\author{
J. H. Ali and M. Shanshal \\ Department of Chemistry, College of Science, Sabha University, Sabha-Libya, P.O. Box 743, Sabha, \\ Libya \\ Reprint requests to Dr. J. H. Ali.
}

Z. Naturforsch. 58a, 162 - 166 (2003); received October 1, 2002

The semi-empirical MINDO/3-FORCES method is extended to include the third row elements $\mathrm{Si}$, $\mathrm{P}, \mathrm{S}$ and $\mathrm{Cl}$. The extended method is tested by calculation of heats of formation, dipole moments, and the first ionization potentials of molecules containing $\mathrm{S}$ and $\mathrm{Cl}$. The results agree well with the experimental and theoretical values known in the literature.

Key words: MINDO/3-FORCES; Third Row Elements.

\section{Introduction}

The semi-empirical MINDO/3-FORCES method was applied by $\mathrm{M}$. Shanshal et al. [1-3]. In this method the molecular energy, obtained with the MINDO/3 method suggested by Dewar et al. [4] was completely minimized with the Murtagh-Sargent minimization technique [5]. The derivative of the energy was calculated analytically with Pulay's Force method [6]. The MINDO/3-FORCES method was used to reproduce the following ground state properties: Molecular geometries [1,7-10], heats of formation [1,7-10], dipole moments [7-9], ionization potentials [7-9], vibrational frequencies, and infrared absorption intensities $[11-13]$. On the other hand, the MINDO/3-FORCES method was restricted to the first and second row elements $\mathrm{H}, \mathrm{B}, \mathrm{C}, \mathrm{N}, \mathrm{O}, \mathrm{F}$ [1]. In the present paper the MINDO/3-FORCES method is extended to the third row elements $\mathrm{Si}, \mathrm{P}, \mathrm{S}, \mathrm{Cl}$, and the validity of this method is examined for molecules containing $\mathrm{S}$ and $\mathrm{Cl}$.

\section{The Extension Procedure}

The extension of the MINDO/3-FORCES method to include third row elements is summarized as follows:

2.1. The calculation of the two center overlap integrals involved the application of the overlap integral formulas given by Mullikan et al. [14] for $1 \mathrm{~s}, 2 \mathrm{~s}, 3 \mathrm{~s}$, $2 \mathrm{p}, 3 \mathrm{p}$ atomic orbitals in the extended overlap subrou- tine [13]. The the analytical derivative of each overlap is calculated. The overlap integrals, $\mathrm{S}$, and their derivatives, $\mathrm{SD}$, are described as follows:

$$
\begin{aligned}
& Z_{1}=.5\left(Z_{\mathrm{A}}+Z_{\mathrm{B}}\right), \\
& Z_{2}=.5\left(Z_{\mathrm{A}} \cdot Z_{\mathrm{B}}\right), \\
& R=R_{1} / .529167, \\
& P=Z_{1} R \\
& Q=Z_{2} R \\
& T=Z_{2} / Z_{1},
\end{aligned}
$$

where $Z_{\mathrm{A}}$ and $Z_{\mathrm{B}}$ are the Slater orbital exponents of atom $\mathrm{A}$ and $\mathrm{B}$, respectively. $R_{1}$ is the interatomic distance between $\mathrm{A}$ and $\mathrm{B}$. The $A(k)$ and $B(k)$, see below, are functions of the Slater orbital exponents used in evaluating the overlap integrals.

First Row-Third Row:

$$
\begin{aligned}
P_{1}= & P^{5}\left(1-T^{2}\right)^{7 / 2} \\
\mathrm{~S}(1 \mathrm{~s}, 3 \mathrm{~s})=.0132 P_{1} & {[A(4) B(0)-B(4) A(0)} \\
& -2\{A(3) B(1)-B(3) A(1)\}],
\end{aligned}
$$




$$
\begin{array}{r}
\mathrm{SD}(1 \mathrm{~s}, 3 \mathrm{~s})=\frac{5 S}{R}-P_{1}\left[Z_{1}\{A(5) B(0)-B(4) A(1)-2(A(4) B(1)-B(3) A(2))\}\right. \\
\left.+Z_{2}\{A(4) B(1)-B(5) A(0)-2(A(3) B(2)-B(4) A(1))\}\right] \\
\begin{array}{r}
\mathrm{S}\left(1 \mathrm{~s}, 3 \mathrm{p}_{\sigma}\right)=.0228 P_{1}[A(3)(B(0)+B(2))+B(3)(A(0)+A(2))-B(1)(A(2)+A(4))-A(1)(B(2)+B(4))] \\
\mathrm{SD}\left(1 \mathrm{~s}, 3 \mathrm{p}_{\sigma}\right)=\frac{5 S}{R}-P_{1}\left[Z_{1}\{A(4)(B(0)+B(2))+B(3)(A(1)+A(3))-B(1)(A(3)+A(5))\right. \\
-A(2)(B(2)+B(4))+Z_{2}\{A(3)(B(1)+B(3))+B(4)(A(0)+A(2)) \\
-B(2)(A(2)+A(4))-A(1)(B(3)+B(5))\}]
\end{array}
\end{array}
$$

Second Row-Third Row:

$$
\begin{aligned}
& P_{1}=P^{6}(1-T)\left(1-T^{2}\right)^{5 / 2} \\
& \mathrm{~S}(2 \mathrm{~s}, 3 \mathrm{~s})=.0038 P_{1}[A(5) B(0)-A(4) B(1)-2\{A(3) B(2)-A(2) B(3)\}+A(1) B(4)-A(0) B(5)], \\
& \mathrm{SD}(2 \mathrm{~s}, 3 \mathrm{~s})=\frac{6 S}{R}-P_{1}\left[Z_{1}\{A(6) B(0)-A(5) B(1)-2(A(4) B(2)-A(3) B(3))+A(2) B(4)-A(1) B(5)\}\right. \\
& \left.+Z_{2}\{A(5) B(1)-A(4) B(2)-2(A(3) B(3)-A(2) B(4))+A(1) B(5)-A(0) B(6)\}\right] \\
& \mathrm{S}\left(2 \mathrm{~s}, 3 \mathrm{p}_{\sigma}\right)=.0066 P_{1}[-A(5) B(1)+A(4) B(0)+2(A(3) B(3)-A(2) B(2))-A(1) B(5)+A(0) B(4)] \text {, } \\
& \mathrm{SD}\left(1 \mathrm{~s}, 3 \mathrm{p}_{\sigma}\right)=\frac{6 S}{R}-P_{1}\left[Z_{1}\{A(6)(B(1)+(A(5) B(0)+2(A(4) B(3)-A(3) B(2))-A(2) B(5)+A(1) B(4)\}\right. \\
& +Z_{2}(-A(5) B(2)+A(4) B(1)+2(A(3) B(4)-(A(2) B(3))-A(1) B(6)+A(0) B(5)\}] . \\
& \mathrm{S}\left(2 \mathrm{p}_{\sigma}, 3 \mathrm{~s}\right)=.0066 P_{1}[-A(4)\{2 B(2)-B(0)\}+B(4)\{2 A(2)-A(0)\} \\
& -A(1)\{B(5)-2 B(3)\}+B(1)\{A(5)-2 A(3)\}], \\
& \mathrm{S}\left(2 \mathrm{p}_{\sigma}, 3 \mathrm{~s}\right)=.0066 P_{1} \frac{6 S}{R}-P_{1}\left[Z_{1}\{-A(5)(2 B(2)-B(0))+B(4)(2 A(3)-A(1))-A(2)(B(5)-2 B(3))\right. \\
& +B(1)(A(6)-2 A(4))\}+Z_{2}\{-A(4)(2 B(3)-B(1)) \\
& +B(5)(2 A(2)-A(0))-A(1)(B(6)-2 B(4))+B(2)(A(5)-2 A(3))\}],
\end{aligned}
$$




$$
\begin{aligned}
\mathrm{S}\left(2 \mathrm{p}_{\sigma}, 3 \mathrm{p}_{\sigma}\right)=-.0114 P_{1}[ & -B(3)(A(0)+A(4))-A(3)(B(0)(B(4)+B(4)) \\
& +B(2)(A(1)+A(5))+A(2)(B(1)+B(5))],
\end{aligned}
$$

$\mathrm{SD}\left(2 \mathrm{p}_{\sigma}, 3 \mathrm{p}_{\sigma}\right)=\frac{6 S}{R}-P_{1}\left[Z_{1}\{-B(3)(A(1)+A(5))-A(4)(B(0)+B(4))+B(2)(A(2)+A(6))\right.$

$$
\begin{aligned}
& +A(3)(B(1)+B(5))\}+Z_{2}\{-B(4)(A(0)+A(4))-A(3)(B(1)+B(5)) \\
& +B(3)(A(1)+A(5))+A(2)(B(2)+B(6))\}]
\end{aligned}
$$

$\mathrm{SD}\left(2 \mathrm{p}_{\pi}, 3 \mathrm{p}_{\pi}\right)=.0057 P_{1}[A(5)\{B(0)-B(2)\}+B(5)\{A(0)-A(2)\}-A(4)\{B(1)-B(3)\}$

$$
-B(4)\{A(1)-A(3)\}-A(3)(B(0)-B(3) A(0)+A(2) B(1)+B(2) A(1)],
$$

$\mathrm{SD}\left(2 \mathrm{p}_{\pi}, 3 \mathrm{p}_{\pi}\right)=\frac{6 S}{R}-P_{1}\left[Z_{1}\{A(6)(B(0)-B(2))+B(5)(A(1)-A(3))-A(5)(B(1)-B(3))\right.$

$$
\begin{aligned}
&-B(4)(A(2)-A(4))-A(4) B(0)-B(3) A(1)+A(3) B(1)+B(2) A(2)\} \\
&+Z_{2}\{A(5)(B(1)-B(3))+B(6)(A(0)-A(2))-A(4)(B(2)-B(4)) \\
&\quad-B(5)(A(1)-A(3))-A(3) B(1)-B(4) A(0)+A(2) B(2)+B(3) A(1)\}] .
\end{aligned}
$$

Third Row-Third Row:

$$
\begin{aligned}
& P_{1}=P^{7}\left(1-T^{2}\right)^{7 / 2}, \\
& \mathrm{~S}(3 \mathrm{~s}, 3 \mathrm{~s})=.0007 P_{1}[A(6) B(0)-3\{A(4) B(2)-A(2) B(4)\}-A(0) B(6)] \text {, } \\
& \mathrm{SD}(3 \mathrm{~s}, 3 \mathrm{~s})=\frac{7 S}{R}-P_{1}\left[Z_{1}\{-A(7) B(0)-3(A(5) B(2)-A(3) B(4))-A(1) B(6)\}\right. \\
& \left.+Z_{2}\{-A(6) B(1)-3(A(4) B(3)-A(2) B(5))-A(0) B(7)\}\right], \\
& \mathrm{S}\left(3 \mathrm{~s}, 3 \mathrm{p}_{\sigma}\right)=.0012 P_{1}[A(5)(B(0)-B(2))-2 A(3)(B(2)-B(4)+A(1))(B(4)-B(6)) \\
& +B(5)(A(0)-A(2))-2 B(3)(A(2)-A(4))-B(1)(-A(4)+A(6))], \\
& \mathrm{SD}\left(3 \mathrm{~s}, 3 \mathrm{p}_{\sigma}\right)=\frac{7 S}{R}-P_{1}\left[Z_{1}\{A(6)(B(0)-B(2))-2 A(4)(B(2)-B(4))+A(2)(B(4)-B(6))\right. \\
& +B(5)(A(1)-A(3))-2 B(3)(A(3)-A(5))+B(1)(-A(5)+A(7))\} \\
& +Z_{2}\{A(5)(B(1)-B(3))-2 A(3)(B(3)-B(5))+A(1)(B(5)-B(7)) \\
& +B(6)(A(0)-A(2))-2 B(4)(A(2)-A(4))-B(2)(-A(4)+A(6))\}],
\end{aligned}
$$


Table 1. Comparison of our MINDO/3-FORCES's results (third rows) with Dewar's MINDO/3 [15] ones (second rows) and the observed [15] ones (first rows).

\begin{tabular}{|c|c|c|c|c|c|c|c|}
\hline Compound & $\begin{array}{c}\Delta H_{f} \\
\mathrm{~kJ} / \mathrm{mole}\end{array}$ & $\begin{array}{l}\text { Dipole moment } \\
\text { (D) }\end{array}$ & $\begin{array}{c}\text { Ionization } \\
\text { potential }\end{array}$ & Compound & $\begin{array}{c}\Delta H_{f} \\
\mathrm{~kJ} / \mathrm{mole}\end{array}$ & $\begin{array}{l}\text { Dipole moment } \\
\text { (D) }\end{array}$ & $\begin{array}{r}\text { Ionization } \\
\text { potential }\end{array}$ \\
\hline \multirow{3}{*}{$1-\mathrm{CH}_{3} \mathrm{SH}$} & -22.1 & - & 9.44 & \multirow{3}{*}{$2-\mathrm{CCL}_{4}$} & -102.8 & 0.003 & 11.47 \\
\hline & -22.5 & - & 9.93 & & -101.5 & 0 & 11.32 \\
\hline & -33.0 & 2.857 & 9.52 & & -102.8 & 0 & 11.32 \\
\hline \multirow{3}{*}{$3-\mathrm{CL}_{2}$} & 0 & 0 & 11.63 & \multirow{3}{*}{$4-S_{2}$} & - & - & \\
\hline & 0 & 0 & 10.82 & & 204.8 & - & 8.36 \\
\hline & -.5 & 0 & 10.81 & & 202.3 & - & 8.40 \\
\hline \multirow{3}{*}{$5-\mathrm{CS}_{2}$} & 117.0 & 0 & 10.08 & \multirow{3}{*}{$6-\mathrm{H}_{2} \mathrm{~S}$} & -20.5 & - & 10.48 \\
\hline & 113.7 & 0 & 9.68 & & -7.9 & - & 9.9 \\
\hline & 112.9 & 0 & 9.7 & & -12.1 & 3.23 & 10.8 \\
\hline \multirow{3}{*}{$7-\mathrm{HCl}$} & -92.4 & 1.08 & 12.8 & \multirow{3}{*}{$8-\mathrm{CH}_{3} \mathrm{Cl}$} & -80.7 & 1.87 & 11.26 \\
\hline & -89.9 & 1.71 & 12.11 & & -63.9 & 1.62 & 11.11 \\
\hline & -89.9 & 1.66 & 12.10 & & -63.9 & 1.55 & 11.10 \\
\hline \multirow{3}{*}{$9-\mathrm{C}_{6} \mathrm{H}_{6} \mathrm{Cl}$} & -52.8 & 1.69 & 9.70 & \multirow{3}{*}{$10-\mathrm{C}_{6} \mathrm{H}_{6} \mathrm{SH}$} & 12.4 & - & - \\
\hline & -84.0 & 1.96 & 9.08 & & 95.3 & - & 8.48 \\
\hline & -84.4 & 1.90 & 9.08 & & 92.4 & 2.78 & 8.48 \\
\hline
\end{tabular}

$$
\begin{aligned}
& \mathrm{S}\left(3 \mathrm{p}_{\sigma}, 3 \mathrm{p}_{\sigma}\right)=.0021 P_{1}[A(2)(B(6)-2 B(2))-A(4)(B(0)+2 B(4))-B(4) A(0)+A(6) B(2)], \\
& \mathrm{SD}\left(3 \mathrm{p}_{\sigma}, 3 \mathrm{p}_{\sigma}\right)=\frac{7 S}{R}-P_{1}\left[Z_{1}\{A(3)(B(6)+2 B(2))-A(5)(B(0)+2 B(4))-B(4) A(1)+A(7) B(2)\}\right. \\
& \left.+Z_{2}\{A(2)(B(7)+2 B(3))-A(4)(B(1)+2 B(5))-B(5) A(0)+A(6) B(3)\}\right], \\
& \mathrm{S}\left(3 \mathrm{p}_{\Pi}, 3 \mathrm{p}_{\Pi}\right)=.0021 P_{1}[A(6)(B(0)-B(2))+B(6)(A(0)-A(2))+A(4)(B(4)-B(2)-B(0)) \\
& +B(4)(A(4)-A(2)-A(0))+2 A(2) B(2)], \\
& \mathrm{SD}\left(3 \mathrm{p}_{\Pi}, 3 \mathrm{p}_{\Pi}\right)=\frac{7 S}{R}-P_{1}\left[Z_{1}\{A(7)(B(0)-B(2))+B(6)(A(1)-A(3))+A(5)(B(4)-B(2)-B(0))\right. \\
& +B(4)(A(5)-A(3)-A(1))+2 A(3) B(3)\} \\
& +Z_{2}\{A(6)(B(1)-B(3))+B(7)(A(0)-A(2))+A(4)(B(5)-B(3)-B(1)) \\
& +B(5)(A(4)-A(2)-A(0))+2 A(2) B(3)\}] \text {. }
\end{aligned}
$$

2.2. The MINDO/3-FORCES's FORTRAN program, used in this study, consists of more than twenty subroutines [10] and among them the OVERLAP, ZOTZ, and FORCE subroutines should be extended [13].

2.3. Introduction the numerical data corresponding the parameters required for the atoms of third row elements (Si, P, S, Cl) [4].

\section{Results and Discussion}

For testing the validity of the extended MINDO/3FORCES method, in Table 1 the calculated heats of formations, dipole moments and ionization energies of ten molecules containing $\mathrm{S}$ and $\mathrm{Cl}$ atoms are compared with those observed [15] and with Dewar's MINDO/3 
results [15]. The agreement is very good, and in some cases our calculated values are nearer to the observed ones. The difference between our results and the corresponding Dewar-ones [15] is due to the different min- imization procedure. In the MINDO/3-method [15], the Davidon-Fletcher-Powell mimimization procedure [16] is used, whereas the MINDO/3-FORCES method uses the Murtagh-Sargent minimization technique [5].
[1] S. M. Khalil and M. Shanshal, Theoret. Chim. Acta (Berl.) 46, 23 (1977).

[2] S. M. Khalil and M. Shanshal, Z. Naturforsch. 33a, 722 (1978).

[3] R. I. Al-Niami and M Shanshal, Z. Naturforsch. 35a, 129 (1978).

[4] R. C. Bingham, M. J. S. Dewar, and D. H. Lo, J. Amer. Chem. Soc. 97, 1285 (1975).

[5] B. A. Murtagh and R. W. H. Sargent, Comput. J. 13, 185 (1970).

[6] P. Pulay, Molec. Phys., 17, 197 (1969).

[7] S. M. Khalil, Z. Naturforsch. 40a, 1278 (1985).

[8] S. M. Khalil, Z. Naturforsch. 43a, 485 (1988).

[9] S. M. Khalil and H. M. Jarjis, Z. Naturforsch. 46a, 247 (1985).
[10] D. H. Abed, M. Sc. thesis, College of Science, Baghadad University (1981).

[11] D. H. Abed, Ph. D. thesis, College of Science, Baghadad University (1987).

[12] D. H. Abed, S. F. Al-Seaidi, and M. Shanshal, Chimica Acta Turc., 7, 23 (1995).

[13] J. H. Ali, Ph. D. thesis, College of Science, Saddam University (1995).

[14] R. S. Mullikan, C. A. Pleke, O. Orloff, and H. Orloff, J. Chem. Phys. 17, 1248 (1949).

[15] M. J. S. Dewar, D. H. Lo, and C. A. Ramsden, J. Amer. Chem. Soc. 97, 1311 (1975).

[16] W. C. Davidon, Comput. J. 10, 406 (1968); R. Fletcher, Comput. J. 8, 33 (1965); R. Fletcher and M. J. D. Powell, Comput. J. 6, 163 (1963). 